\title{
NÍVEIS DE RUÍDO EMITIDOS POR TRATORES AGRÍCOLAS
}

\author{
M. M. Baesso*, A. J. Modolo, R. C. E. Baesso, C. Fischer
}

USP - Univ de São Paulo, Faculdade de Zootecnia e Engenharia de Alimentos, Campus de Pirassununga, SP, Brasil

Article history: Received 04 July 2017; Received in revised form 28 August 2017; Accepted 30 August 2017; Available online 29 September 2017.

\section{RESUMO}

A exposição do operador ao ruído emitido pelos tratores agrícolas pode ser prejudicial à saúde. Este trabalho foi conduzido com o objetivo de avaliar os níveis de ruído emitidos por tratores agrícolas de diferentes marcas, potências e ano de fabricação, comparando-se os resultados com as normas vigentes no Brasil. O trabalho, realizado com 22 tratores, foi desenvolvido em propriedades rurais localizadas no município de Pirassununga, SP, e na Faculdade de Zootecnia e Engenharia de Alimentos (FZEA) da Universidade de São Paulo (USP), situada no município de Pirassununga, em área cedida pela Prefeitura Administrativa do Campus USP Fernando Costa de Pirassununga. Os níveis de ruído foram determinados por um medidor de pressão sonora digital, marca ICEL, modelo DL-4020. O Decibelímetro foi ajustado para medir os resultados expressos em $\mathrm{dB}$ (A) nos circuitos de resposta lenta. Observou-se nível de ruído acima do máximo permitido para uma jornada de trabalho de 8 horas em todos os tratores estudados.

Palavras-chave: ergonomia; exposição do operador; segurança no trabalho.

\section{LEVELS OF NOISE EMITTED BY AGRICULTURAL TRACTORS}

\begin{abstract}
The exposure of workers to noise produced by agricultural tractors, which can be harmful to your health. This study aims to evaluate the levels of noise emitted by agricultural tractors of different powers and to compare the results with existing regulations in Brazil. This work, studied 22 tractors, was performed at the farms in Pirassununga, SP and Faculty of Animal Sciences and Food Engineering (FZEA) at the University of São Paulo in Pirassununga County, in an area owned by the Prefecture of the Campus USP Fernando Costa. To measure noise, Icel DL 4020 decibelimeter were used. The decibelimeter scale used were decibels $\mathrm{dB}$ (A), with the instrument adjusted to measure the machine noise with quick answer. Noise level above the maximum allowed for the work day of 8 hours was observed in all tractors studied.
\end{abstract}

Keywords: ergonomic; operator exposure; safety at work. 


\section{INTRODUÇÃO}

Com a modernização da agricultura, observaram-se importantes avanços tecnológicos nos tratores agrícolas, que propiciaram expressiva evolução na exploração agrícola nacional, permitindo aumento na produtividade ao longo dos últimos 40 anos, na grande parte dos cultivos agrícolas.

A preocupação com o conforto e com a segurança do operador tem chamado a atenção de profissionais de diversas áreas, no sentido de considerar os fatores humanos (ergonomia) na concepção projetual de tratores agrícolas, principalmente em razão das adversidades impostas pela natureza no meio agrícola e também pela periculosidade que essas máquinas apresentam e pelos acidentes envolvidos nesse contexto (ANTONUCCI et al., 2012).

A principal preocupação da ergonomia é com o ser humano, sendo seu objetivo a melhor condição do trabalhador, relacionando-se o acréscimo de produção ou melhoria da qualidade dos produtos com os resultados de uma interação adequada entre o homem e o sistema de produção (FILIP; CANDALE, 2012).

Entre os fatores ergonômicos que prejudicam os operadores das máquinas agrícolas, o ruído é um dos principais, com o agravante de existirem poucos trabalhos desenvolvidos para a avaliação desse parâmetro e suas implicações na saúde ocupacional (CUNHA; TEODORO, 2006). Bilski (2013) afirmou que os tratores agrícolas são uma das fontes mais proeminentes de ruído na agricultura, e suas alterações não têm efeitos imediatos e, sim, acumulativos e vão-se implantando com o tempo: hipoacusia, desequilíbrios psíquicos e doenças físicas degenerativas. Poje et al. (2016) destacaram a necessidade de estudar o ruído produzido por máquinas agrícolas, com o objetivo de compreender melhor sua propagação e o efeito causado, principalmente na audição do operador agrícola.

Cunha et al. (2012), estudando níveis de ruídos em dois tratores agrícolas, defasados temporalmente, mostraram que ambos os tratores apresentaram níveis de ruído acima do limite de $85 \mathrm{~dB}$ (A). E ainda afirmaram que a grande evolução dos tratores nos últimos anos não foi capaz de reduzir o ruído a níveis passíveis de trabalho sem protetor auricular nos tratores sem cabina, para uma jornada de oito horas diárias de serviço.

Segundo Menezes e Paulino (2004), a exposição ao ruído pode resultar em perda auditiva temporária ou permanente, sendo a perda auditiva temporária causada por exposições moderadas e é considerada recuperável. A Associação Brasileira de Normas Técnicas (ABNT) estabelece os níveis de ruído para o conforto acústico e para o nível de ruído de $85 \mathrm{~dB}$, e a exposição permitida é de oito horas por dia (BRASIL, 1978).

Vê-se, na Tabela 1, a máxima exposição diária permissível, segundo a Norma Regulamentadora (NR 15) do Ministério do Trabalho e Emprego. 
Tabela 1 - Máxima exposição diária permissível e nível de ruído

\begin{tabular}{cc}
\hline Nível de ruído dB (A) & Máxima Exposição Diária Permissível \\
\hline 85 & $8 \mathrm{~h}$ \\
86 & $7 \mathrm{~h}$ \\
87 & $6 \mathrm{~h}$ \\
88 & $5 \mathrm{~h}$ \\
90 & $4 \mathrm{~h}$ \\
92 & $3 \mathrm{~h}$ \\
95 & $2 \mathrm{~h}$ \\
100 & $1 \mathrm{~h}$ \\
105 & $30 \mathrm{~min}$ \\
110 & $15 \mathrm{~min}$ \\
115 & 7 min \\
\hline
\end{tabular}

Fonte: Norma Regulamentadora (NR 15) do Ministério do Trabalho e Emprego, 1978.

Este trabalho foi conduzido com o objetivo de avaliar os níveis de ruído emitidos por tratores agrícolas de diferentes marcas, potências e ano de fabricação, comparando-se os resultados com as normas vigentes no Brasil.

\section{MATERIAL E MÉTODOS}

O trabalho foi desenvolvido na Faculdade de Zootecnia e Engenharia de Alimentos (FZEA) da Universidade de São Paulo (USP), localizada no município paulista de Pirassununga, em área cedida pela Prefeitura Administrativa do Campus USP Fernando Costa de Pirassununga. A localização geográfica do campus é $21^{\circ} 59^{\prime}$ de latitude Sul e $47^{\circ} 26^{\prime}$ de longitude Oeste e altitude média de 635 metros.

$\mathrm{O}$ ensaio foi realizado com 22 tratores, de diferentes marcas, potências e ano de fabricação: Massey Ferguson MF 265 (potência no motor de $65 \mathrm{cv}$, fabricado no ano 1984), Massey Ferguson MF 65 X (potência no motor de $65 \mathrm{cv}$, fabricado no ano 1974), FORD 4600 (potência no motor de $68 \mathrm{cv}$, fabricado no ano 1977), Massey Ferguson MF 4275 (potência no motor de $75 \mathrm{cv}$, fabricado no ano 2011), Massey Ferguson MF 275 (potência no motor de $75 \mathrm{cv}$, fabricado no ano 1987), Massey Ferguson MF $275 \mathrm{C}$ (potência no motor de $75 \mathrm{cv}$, fabricado no ano 1987), Valmet: 785 (potência no motor de $75 \mathrm{cv}$, fabricado no ano 1994), New Holland TL 80 (potência no motor de $80 \mathrm{cv}$, fabricado no ano 2000), Massey Ferguson MF 290 (potência no motor de $80 \mathrm{cv}$, fabricado no ano 1986), Valmet 88 (potência no motor de $80 \mathrm{cv}$, fabricado no ano 1984), New Holland TL (potência no motor de $90 \mathrm{cv}$, fabricado no ano 2005), Valtra BL 88 (potência no motor de $90 \mathrm{cv}$, fabricado no ano 2005), Massey Ferguson MF 292 (potência no motor de $97 \mathrm{cv}$, fabricado no ano 1989), Massey Ferguson MF 4292 (potência no motor de $110 \mathrm{cv}$, fabricado no ano 2011), Valtra BM 110 (potência no motor de $110 \mathrm{cv}$, fabricado no ano 2013), John Deere 9110J (potência no motor de $110 \mathrm{cv}$, fabricado no ano 2013), Massey Ferguson MF 297 (potência no motor de $120 \mathrm{cv}$, fabricado no ano 2005), Valtra BM 125 (potência no motor de $125 \mathrm{cv}$, fabricado no ano 2011), Valtra BM 125i C (potência no motor de $130 \mathrm{cv}$, fabricado no ano 2010), Valtra BH 145 (potência no motor de $145 \mathrm{cv}$, fabricado no ano 2012), Massey Ferguson MF 680 (potência no motor de $173 \mathrm{cv}$, fabricado no ano 1997) e Valtra BT 210 C (potência no motor de $215 \mathrm{cv}$, fabricado no ano 2012).

$\mathrm{Na}$ Tabela 2, apresentam-se o modelo, a potência e o ano de fabricação dos tratores estudados. 
Tabela 2 - Modelo, potência e o ano de fabricação dos tratores estudados

\begin{tabular}{ccc}
\hline Modelo & $\begin{array}{c}\text { Potência } \\
\text { (cv) }\end{array}$ & Ano de fabricação \\
\hline Massey Ferguson 265 & 65 & 1984 \\
\hline Massey Ferguson 65 X & 65 & 1974 \\
\hline Ford 4600 & 68 & 1977 \\
\hline Massey Ferguson 4275 & 75 & 2011 \\
\hline Massey Ferguson 275 & 75 & 1987 \\
\hline Massey Ferguson 275 C & 75 & 1987 \\
\hline Valmet 785 & 75 & 1994 \\
\hline New Holland TL 80 & 80 & 2000 \\
\hline Massey Ferguson 290 & 80 & 1986 \\
\hline Valmet 88 & 80 & 1984 \\
\hline New Holland TL & 90 & 2005 \\
\hline Valtra BL 88 & 90 & 2005 \\
\hline Massey Ferguson 292 & 97 & 1989 \\
\hline Massey Ferguson 4292 & 110 & 2011 \\
\hline Valtra BM 110 & 110 & 2013 \\
\hline John Deere 6110J & 110 & 2013 \\
\hline Massey Ferguson 297 & 120 & 2005 \\
Valtra BM 125 & 125 & 2011 \\
\hline Valtra BM 125i C & 130 & 2010 \\
Valtra BH 145 & 145 & 2012 \\
\hline Massey Ferguson 680 & 173 & 1997 \\
Valtra BT 210 C & 215 & 2012 \\
\hline
\end{tabular}

Os níveis de ruído foram determinados por um medidor de pressão sonora digital, marca ICEL, modelo DL4020, nos circuitos de resposta lenta e de equalização "A", sendo expressos em dB (A). Em todas as medições, foi usado o protetor de vento do medidor de pressão. As leituras foram tomadas próximas ao ouvido do operador (o aparelho foi colocado a $2 \mathrm{~cm}$ do ouvido nos lados esquerdo e direito), e em cada raio de afastamento de um a 10 metros, coletadas de um em um metro, direcionadas para os lados direito e esquerdo e partes traseira e frontal do trator.

Para este estudo, a leitura do ponto foi considerada com base na média de cinco repetições por leitura (ponto amostral). No intuito de facilitar a discussão sobre os raios de afastamento, os tratores foram divididos em três grupos: tratores sem cabina e com ano de fabricação igual ou inferior a 1999, tratores cabinados com fabricação entre os anos 2000 e 2009 e, por fim, tratores cabinados com ano de fabricação igual ou superior a 2010.

As avaliações seguiram conforme descrito na NBR-9999 (ABNT, 1987). Segundo essa norma, na posição e momento do ensaio de medição do nível de ruído, a temperatura ambiente deve estar entre -5 e $30^{\circ} \mathrm{C}$ e a velocidade do vento, ser inferior a $5,0 \mathrm{~m} \mathrm{~s}^{-1}$. Tais condições foram medidas e estavam satisfatórias no momento da avaliação.

\section{RESULTADOS E DISCUSSÃO}

A Tabela 3 apresenta os valores médios dos níveis de ruídos emitidos próximos ao ouvido do operador. 
Tabela 3 - Valores médios dos níveis de ruídos $(\mathrm{dB})$ emitidos próximos ao ouvido do operador

\begin{tabular}{|c|c|c|c|c|}
\hline Modelo & Direito dB (A) & Esquerdo dB (A) & Pot. (cv) & Ano \\
\hline Massey Ferguson 265 & 86,3 & 85,7 & 65 & 1984 \\
\hline Massey Ferguson 65 X & 87,9 & 88,2 & 65 & 1974 \\
\hline Ford 4600 & 92,5 & 92,8 & 68 & 1977 \\
\hline Massey Ferguson $4275^{*}$ & 83,4 & 84,2 & 75 & 2011 \\
\hline Massey Ferguson 275 & 96,1 & 97,2 & 75 & 1987 \\
\hline Massey Ferguson 275** & 86,9 & 87,1 & 75 & 1987 \\
\hline Valmet 785 & 96,8 & 96,7 & 75 & 1994 \\
\hline New Holland TL 80* & 82,2 & 81,2 & 80 & 2000 \\
\hline Massey Ferguson 290 & 88,7 & 88,4 & 80 & 1986 \\
\hline Valmet 88 & 96,2 & $\mathbf{9 7 , 9}$ & 80 & 1984 \\
\hline New Holland TL* & 81,2 & 83,4 & 90 & 2005 \\
\hline Valtra BL $88^{* *}$ & 93,4 & 95,2 & 90 & 2005 \\
\hline Massey Ferguson 292 & 89,7 & 90,3 & 97 & 1989 \\
\hline Massey Ferguson 4292* & 83,4 & 84,8 & 110 & 2011 \\
\hline Valtra BM 110* & 83,1 & 81,9 & 110 & 2013 \\
\hline John Deere 6110J* & 82,9 & 83,7 & 110 & 2013 \\
\hline Massey Ferguson 297* & 84,5 & 84,9 & 120 & 2005 \\
\hline Valtra BM 125* & 90,7 & 91,2 & 125 & 2011 \\
\hline Valtra BM 125i* & 83,2 & 84,7 & 130 & 2010 \\
\hline Valtra BH $145^{*}$ & 83,9 & 82,1 & 145 & 2012 \\
\hline Massey Ferguson 680 & 96,2 & 95,4 & 173 & 1997 \\
\hline Valtra BT 210 C* & 82,1 & 83,5 & 215 & 2012 \\
\hline
\end{tabular}

Entre os 22 modelos de tratores estudados, apenas 10 apresentaram nível de ruído aceitável em relação à NR 15 (abaixo dos $85 \mathrm{~dB}(\mathrm{~A})$ ) próximo ao ouvido do operador para uma jornada de oito horas de trabalho diário. Destaca-se o fato de que todos possuem cabina fechada, demonstrando a sua eficiência. Os demais tratores apresentaram nível de ruído acima de $85 \mathrm{~dB}$ (A) no posto de operação. Resultados semelhantes foram encontrados por Tosin et al. (2009), que, avaliando o nível de ruído em dois tratores agrícolas, um de 55,9 kW sem cabina e outro de 80,9 $\mathrm{kW}$ cabinado, concluíram que a cabina proporciona isolamento acústico, independentemente da potência do motor.

Os modelos Massey Ferguson 275 e Valtra BL 88, apresentaram valores acima do tolerável para uma jornada diária de oito horas, ambos possuíam cabina não original de fábrica, o que possivelmente influenciou diretamente no desempenho acústico. Portanto, é necessário o uso de protetores auriculares por parte do operador desses tratores, mesmo com a cabina fechada. A preocupação com esse tipo de adaptação é discutida em alguns trabalhos, como é o caso de Shrivastava et al. (2014), que, avaliando o nível de ruído em motores agrícolas, propuseram um novo desenho de silenciadores, no intuito de melhorar a eficiência do sistema homem-máquina, principalmente em motores de média e de grande potência.

Foi perceptível maior nível de ruído emitido pelos tratores mais antigos, que em sua maioria não possuem cabina fechada e designs modernos. Cunha et al. (2012) comentaram que o avanço tecnológico é 
capaz de melhorar a saúde, segurança, conforto e eficiência do operador, pois em geral são ergonomicamente mais eficientes.

Em relação ao raio de afastamento, os valores maiores de ruído ocorrem, em geral, até cinco metros de distância do trator, ou seja, para pessoas que estejam numa distância de até cinco metros da maioria dos tratores estudados, durante a sua jornada de trabalho de oito horas, terão que usar equipamento de proteção individual (EPI). Pimenta Junior et al. (2012) também estipularam um raio de cinco metros como indicado para uso de EPI em operações rotineiras com tratores agrícolas, tanto para operadores quanto para os demais funcionários que trabalham em suas proximidades.

A Figura 1 ilustra os resultados obtidos pelos tratores FORD 4600 e Massey Ferguson 65 X, ambos sem cabina fechada e com ano de fabricação igual ou inferior a 1999.

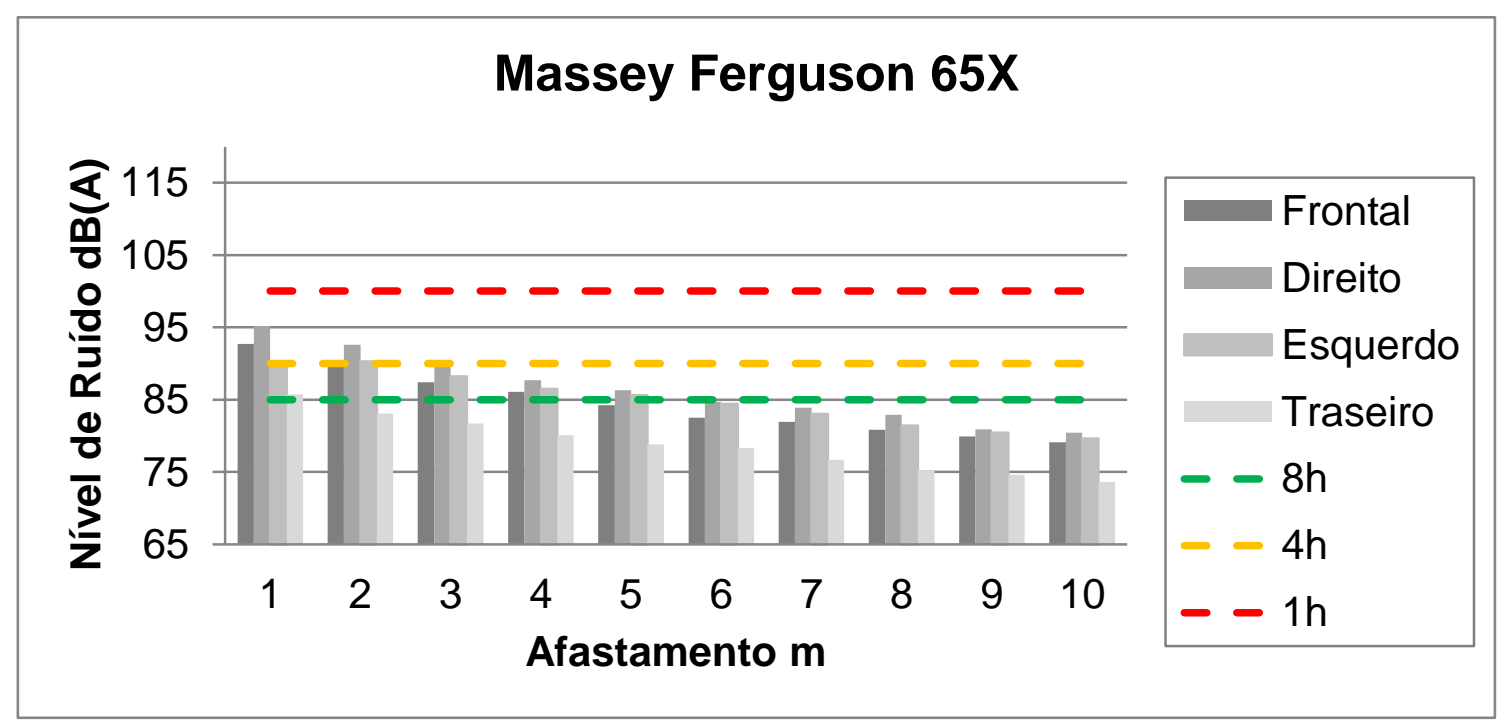

(a)

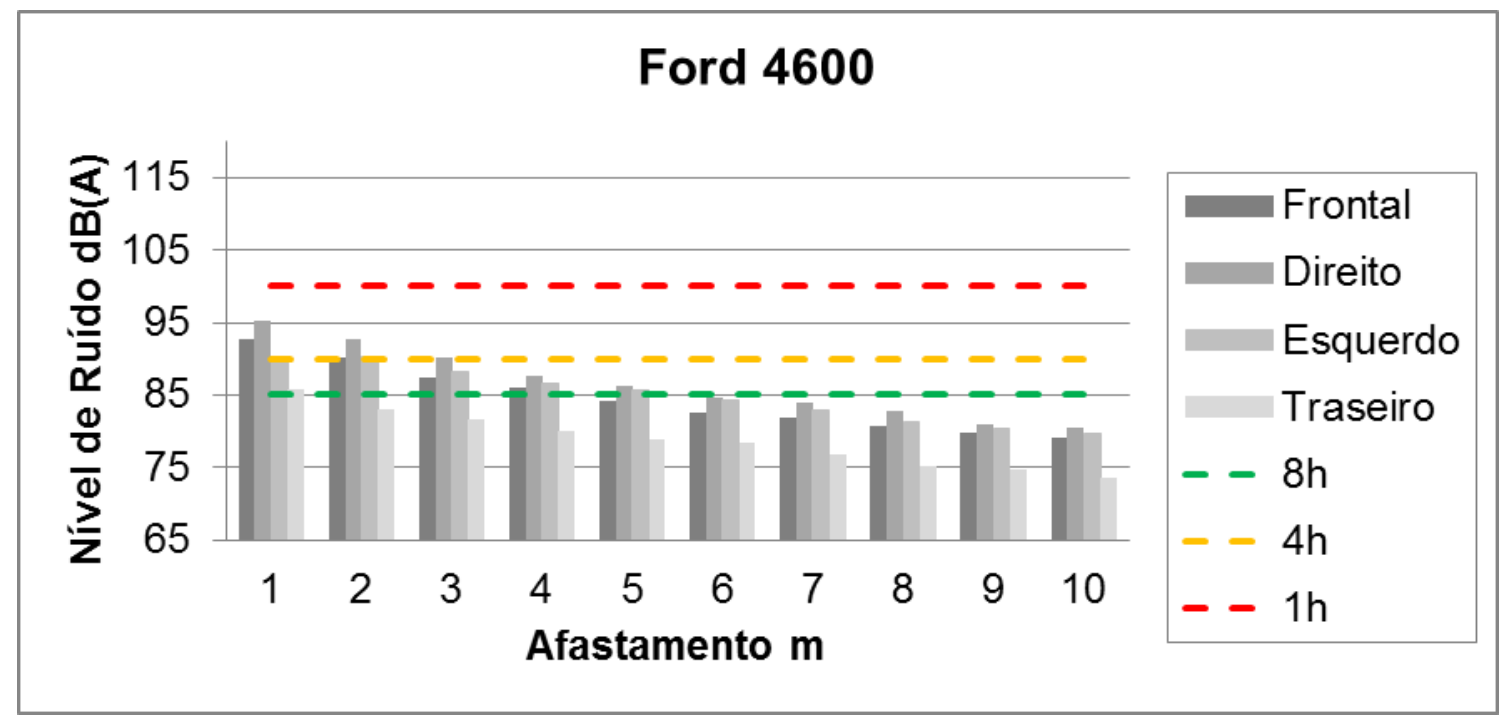

(b)

Figura 1 - Nível de ruído em função do raio de afastamento para os modelos FORD 4600 (a) e Massey Fergunson 65X (b), em todas as direções (frontal, traseira e laterais esquerda e direita). 
Nota-se também, nessa Figura que os dois tratores apresentaram valores de ruídos menores na parte traseira, se comparados com as demais posições (frontal e lateral), possivelmente explicados pelo posicionamento do motor. Os outros tratores pertencentes a esse grupo - Valmet 785, Massey Fergunson 290, Valmet 88, Massey Fergunson 680, Massey Fergunson 275 e Massey Fergunson 292 - apresentaram resultados semelhantes, ou seja, pessoas que estejam numa distância de até cinco metros desses tratores necessitam usar protetor auricular.

Dentro dessa categoria (tratores sem cabina fechada e com ano de fabricação igual ou inferior a 1999), o único trator que apresentou nível de ruído abaixo do limite para uma jornada diária de oito horas, em todos os raios de afastamento estudados, foi o trator Massey Fergunson 265, cujo resultado é ilustrado na Figura 2.

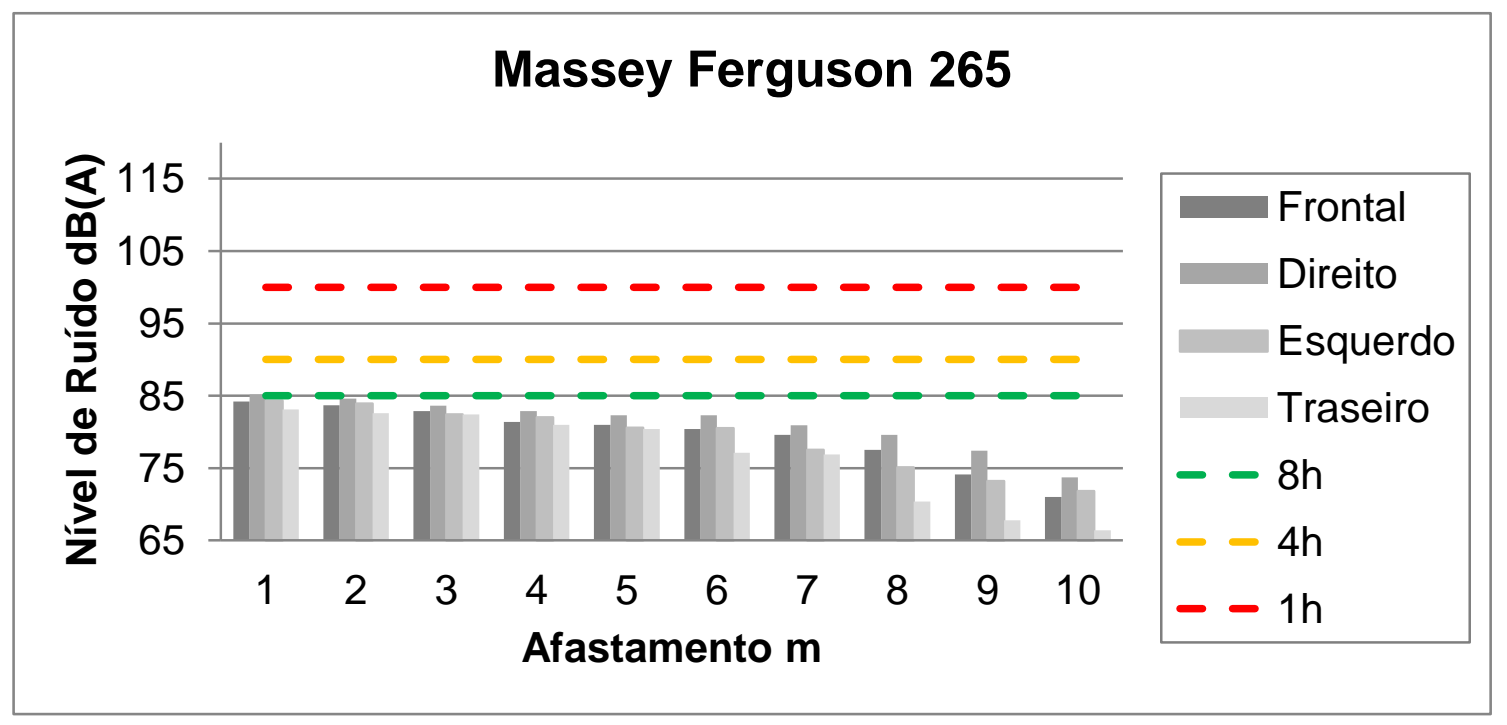

Figura 2 - Nível de ruído em função do raio de afastamento para o modelo Massey Fergunson 265, em todas as direções (frontal, traseira e laterais esquerda e direita).

A Figura 3 ilustra os resultados obtidos pelos tratores New Holland TL 80 e Massey Ferguson 297, ambos com cabina fechada, de fabricação entre os anos de 2000 e 2009. 


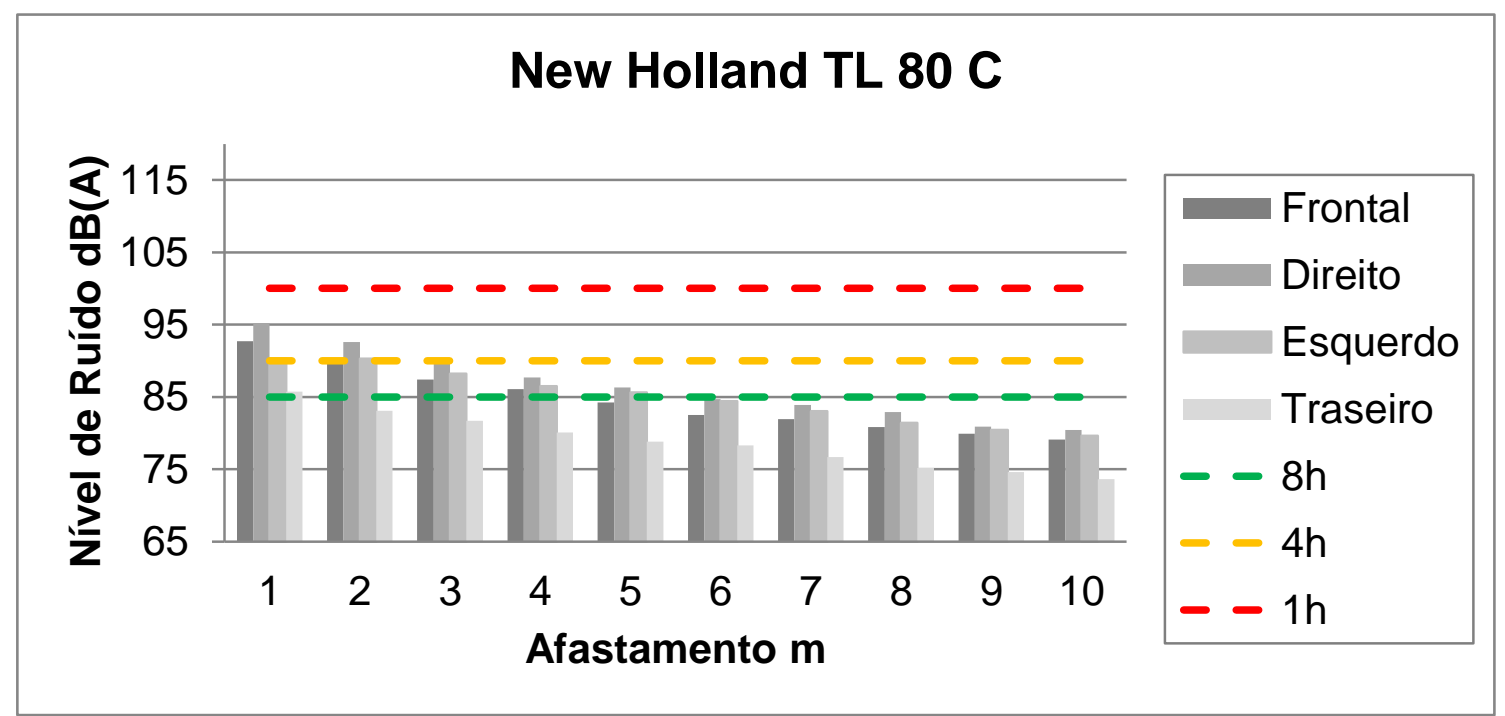

(a)

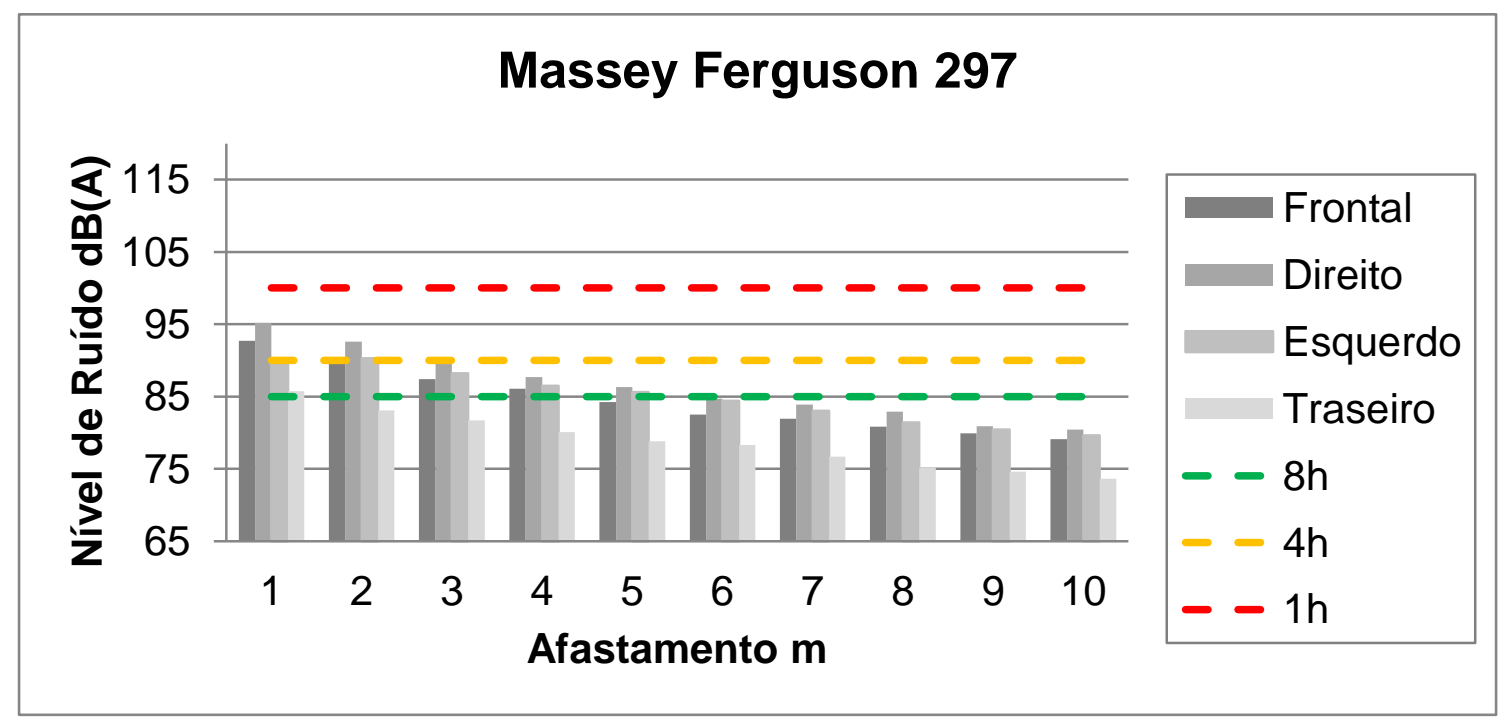

(b)

Figura 3 - Nível de ruído em função do raio de afastamento para os modelos New Holland TL 80 (a) e Massey Ferguson 297 (b), em todas as direções (frontal, traseira e laterais esquerda e direita).

Os demais tratores pertencentes a esse grupo (New Holland TL e Valtra BL 88) apresentaram o mesmo comportamento. Resultados semelhantes foram encontrados por Oliveira Junior et al. (2011) em diferentes raios de afastamento. Os autores concluíram que, mesmo em tratores cabinados, o nível de ruído ao qual pessoas próximas aos tratores estavam expostas estava acima do limite de
$85 \mathrm{~dB}$ (A), principalmente com o aumento da rotação de trabalho do trator.

A Figura 4 ilustra os resultados obtidos pelos tratores Valtra BH 145 e Valtra BT 210, ambos com cabina fechada e com ano de fabricação igual ou superior a 2010. O mesmo comportamento do nível de ruído emitido foi observado nos demais tratores desse grupo (Massey Ferguson 4275, Massey Ferguson 4292, Valtra BM 
110, John Deere 6110J, Valtra BM 125 e Valtra BM 125i).

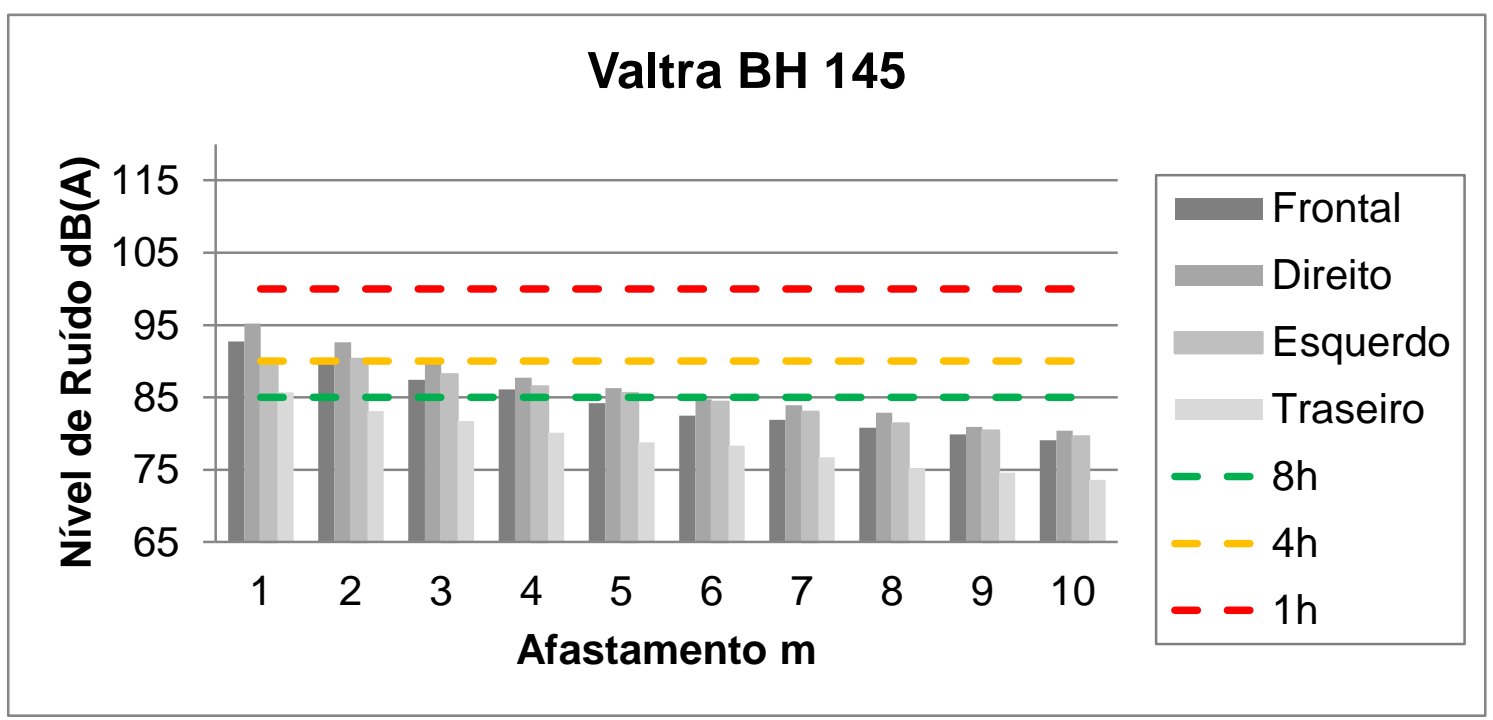

(a)

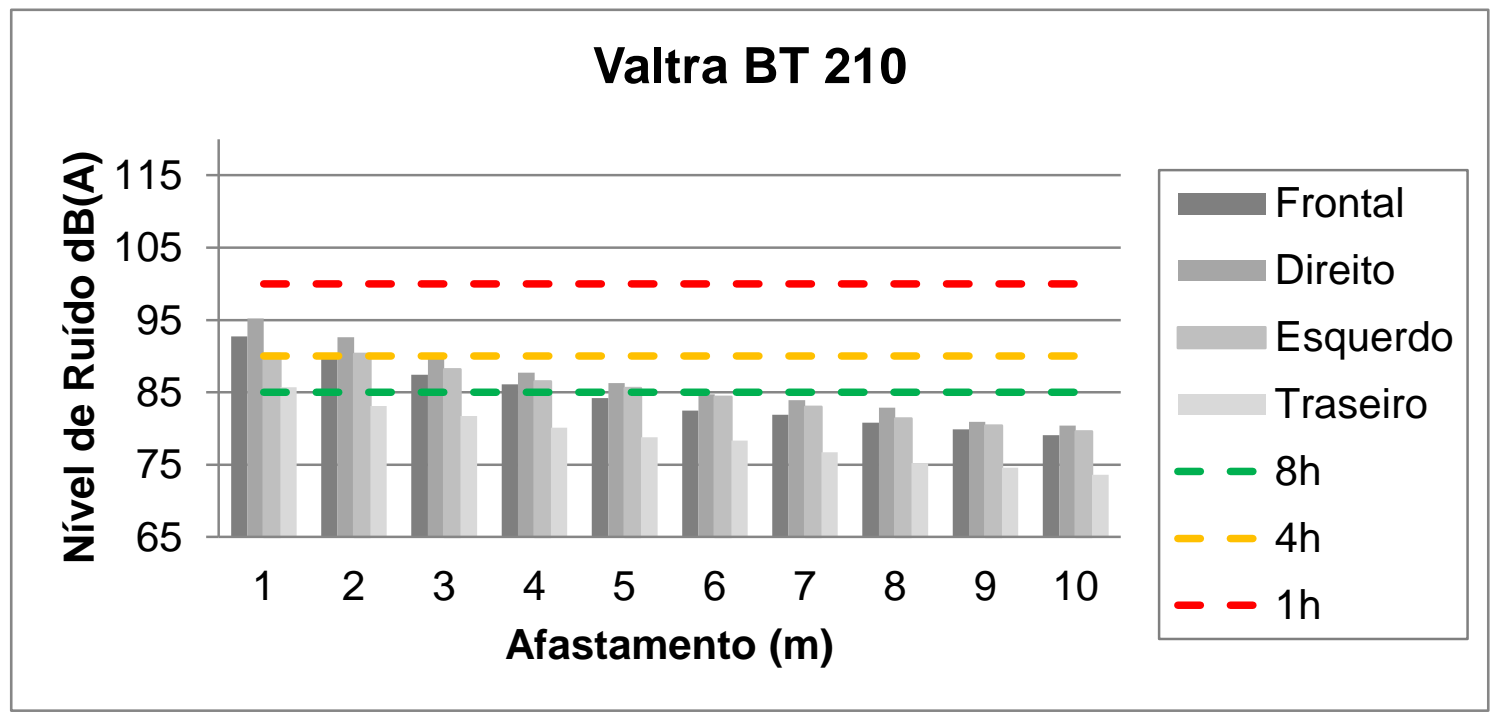

(b)

Figura 4 - Nível de ruído em função do raio de afastamento para os modelos Valtra BH 145 (a) e Valtra BT 210 (b), em todas as direções (frontal, traseira e laterais esquerda e direita).

Observou-se uma relação positiva entre o nível de ruído e a potência do motor, ou seja, maior potência no motor tendeu a apresentar maiores valores de ruído. Em alguns casos o limite máximo permissível aos colaboradores é inferior à

\section{CONCLUSÕES}

Os tratores estudados, em geral, apresentaram níveis de ruído superiores uma hora diária, deixando evidente a necessidade do equipamento de proteção individual (EPI), independente do ano de fabricação, potência do motor e a presença de cabina fechada.

aos estipulados pela Norma Regulamentadora (NR 15) do Ministério 
do Trabalho e Emprego para a jornada de trabalho de oito horas diárias.

Os operadores dos tratores estudados e seus auxiliares estavam sujeitos a risco de hipoacusia quando se trabalha sem proteção auricular.

\section{REFERÊNCIAS}

ANTONUCCI, A. E.; SICILIANO, D.; LADIANA, P.; BOSCOLO, M. S. Perception of occupational risk by rural workers in an area of central italy. Journal of Biological Regulators and Homeostatic Agents, v. 26(3): 439-445, 2012.

ASSOCIAÇÃO BRASILEIRA DE NORMAS TÉCNICAS. NBR 9999: Medição do nível de ruído, no posto de operação de tratores e máquinas

agrícolas. Rio de Janeiro, 1987. 21 p.

BILSKI, B. Exposure to audible and infrasonic noise by modern agricultural tractors operators. Applied Ergonomics, v. 44(1): 210-214, 2013.

BRASIL. Ministério do Trabalho e Emprego. Norma Regulamentadora. NR 15- Atividades e operações insalubres. Portaria MTE n. 3.214, de 8 de junho de 1978. Diário Oficial [da] União, Brasília, 6 Jul. 1978.

CUNHA, J. P. A. R.; DUARTE, M. A. V.; DE SOUZA, C. M. A. Vibração e ruído emitidos por dois tratores agrícolas. Idesia, v. 30(1): 25-34, 2012.

CUNHA, J. P. A. R.; TEODORO, R. E. F. Avaliação do nível de ruído em derriçadores e pulverizadores motorizados portáteis utilizados em lavouras de café. Bioscience Journal, v. 22(3): 71-77, 2006.

FILIP, N.; CANDALE, L. Researches regarding the noise conversion from tractor engine in order to reduce the intake manifold noise. Aktualni Zadaci
Existiu relação positiva entre o nível de ruído e a potência no motor.

Pessoas que estiverem numa distância de até cinco metros durante a sua jornada de trabalho de oito horas devem usar equipamento de proteção individual (EPI).

Mehanizacije Poljoprivrede, v. 40(5): 141-147, 2012.

MENEZES, J. S. R.; PAULINO, N. J. A. Efeitos do ruído no organismo: manual de avaliação e controle do ruído. 3 . ed. São Paulo: LTr, 2004. p. 62-72.

OLIVEIRA JÚNIOR, A.; ALVES, G. S.; CUNHA, J. P. A. R. Avaliação dos níveis de ruído emitido por um trator agrícola em diferentes operações mecanizadas. Enciclopédia Biosfera, v. 7(12), 2011.

PIMENTA JUNIOR, C. G.; DELMOND, J. G.; CUNHA, J. P. B.; COUTO, R. F.; LEONÍDIO, D. M.; REIS, E. F. Análise espacial do nível de ruído emitido por trator agrícola. Revista Brasileira de Ciências Agrárias, v. 7(3): 514-520, 2012. POJE, A.; POTOCNIK, I.; DANILOVIC, M.; ANTONIC, S. A Case Study of the Impact of skidding distance on tractor operator exposure to noise.

Baltic Forestry, v. 22 (2): 357-364, 2016.

SHRIVASTAVA, A. K.; TEWARI, V. K.; SANTOS, H. K. Effect of exhaust back pressure on noise characteristic of tractor mufflers. Agricultural Mechanization in Asia Africa and Latin America, v. 45(1): 79-83, 2014.

TOSIN, R. C. Avaliação do ruído e da vibração no posto de trabalho em dois tratores agrícolas. 2009. 140 f. Tese (Doutorado em Energia na Agricultura) - Faculdade de Ciências Agronômicas, Universidade Estadual Paulista, Botucatu, SP, 2009. 